\title{
Evolution of economic entities under heterogeneous political/environmental conditions within a Bak-Sneppen-like dynamics
}

\author{
Marcel Ausloos ${ }^{1}$, Paulette Clippe ${ }^{2}$ and Andrzej Pȩkalski ${ }^{3}$ \\ ${ }^{1}$ SUPRAS(米 and GRASP, B5, Sart Tilman Campus, \\ B-4000 Liège, Euroland, \\ 2 GRASP, B5, Univ. de Liège, \\ B-4000 Liège, Euroland, \\ ${ }^{3}$ Institute of Theoretical Physics, University of Wrocław, \\ pl. Maxa Borna 9, \\ PL-50-204 Wrocław, Poland
}

November 1, 2018

email:

Marcel.Ausloos@ulg.ac.be, Paulette.Clippe@ulg.ac.be, apekal@ift.uni.wroc.pl

*a member of SUPRATECS 


\begin{abstract}
A model for economic behavior, under heterogeneous spatial economic conditions is developed. The role of selection pressure in a Bak-Sneppen-like dynamics with entity diffusion on a lattice is studied by Monte-Carlo simulation taking into account business rule(s), like enterprise - enterprise short range location "interaction" (s), business plan(s) through spin-offs or merging and enterprise survival evolution law(s). It is numerically found that the model leads to a sort of phase transition for the fitness gap as a function of the selection pressure.
\end{abstract}

Pacs: 89.65.Gh, 05.10.Ln, 89.75.-k, 07.05.Tp, 05.65.+b

Keywords: econophysics, evolution, external field, selection pressure, diffusion process, Bak-Sneppen model, Monte-Carlo

\title{
1 Introduction
}

In a previous paper [1] we have raised the question whether one could through modern econophysics ideas and theories [2] touch upon questions related to changing economic world conditions, e.g. globalization or mondialisation and delocalization, and discuss the consequences of such politics. The Berlin wall destruction and opening of markets in Eastern Europe and Central Asia to so called liberal economy was said to be similar to an increase in "physical volume or available space". The disparity in "economic conditions", like the tax systems, or different workers' skills or wages, weather, available information, ... seemed to be also similar to "external field" conditions in condensed matter.

In a first approach we have asked whether the evolution of the concentration of enterprises, their spatial distribution, their (so we say) "fitness" under varying in time and space economic field conditions can be derived from some microscopic-like model as often found in statistical physics to describe complex phenomena. We have raised the question whether macroeconomy seems to work as a self-organized system [3], characterized by scaling laws, whether models and theories pertaining to such features characterized by punctuated equilibrium contain cycles or stable states $[4,5,6]$.

In [1] we have introduced an economic world model as a lattice divided into $\nu(=3)$ regions of equal size. At the origin of time all "firms" (enter- 
prises, agents, ..) are located only in region I. A barrier preventing companies to enter regions II and III is removed at a given $t_{1}$ time. Each company $(i)$ is characterized by one real number $f_{i}$, belonging to the $[0,1]$ interval. Each region is under some economic conditions, an "external field", represented by one real number, $F$ also belonging to $[0,1]$. The best condition, "by symmetry", is $F=0.5$. We have searched for the evolution of concentrations in regions II and III as invaded according to a simple diffusion rule. A so called business plan, based on a biased merging and spin-off creation alternative was considered for the enterprise evolution. The $f$ value of the new firms was considered to be obtained according to various types of memories depending on the $f$ of the company parents $[1,7]$.

In such a scheme, some attempt was made to connect macroeconomy and econophysics [8] through questions concerning whether enterprises survive or not, get better or worse, how the diffusion front moves toward regions II and III, etc. One ingredient of the Monte-Carlo algorithm was to consider that the evolution was randomly driven, in the sense that the entity picked up by the Monte-Carlo procedure was independent of the position and $f$ value of the firm.

However some other dynamics can be imagined. In particular recall the Bak-Sneppen evolution model [9] which has considered that species being assigned a so called fitness $f$ have a better chance of surviving if $f$ is large. The population evolution is controlled through the entity with the minimal fitness at every discrete time step. This species is replaced by a new one, with a new fitness, taken from a uniform distribution on $[0,1]$. Moreover an interaction is introduced, e.g. by (randomly) modifying the fitness of the neighbors of the chosen entity. This interaction represents a co-evolution of related species. Many variants have been studied, including an anisotropic Bak-Sneppen model [10], a tree growth [11] evolution with or without species screening $[12,13]$, with or without long range interactions [14]... Applications of the Bak-Sneppen ideas occur in many fields, from biological problems, like bacteria colony evolution $[15,16]$ to macro-economical processes $[17,18]$.

We reformulate the simple model presented in [1] for the evolution of economic entities under varying economic conditions introducing a BakSneppen- like dynamics. In Sect. 2, we present the new model algorithm which therefore stresses the role of the selection pressure and the Bak-Sneppenlike dynamics. In section 3, we outline a few results, like the diffusion front penetration, and the concentration of entity evolution. It is numerically ob- 
served that the model leads to a phase transition-like scenario for the fitness gap as a function of the selection pressure. A short conclusion is to be found in Sect. 4.

\section{Model and Monte Carlo Simulation Algo- rithm}

We consider a square symmetry lattice of linear dimensions $L_{x} \times L_{y}$. The $x$ segment is divided into 3 parts (regions I, II and III) of equal size $\left(L_{x}=150\right.$, $L_{y}=201$ ). The entities we consider, called thereafter firms, are located on the lattice sites. A site may be either empty or occupied by one firm.

Each firm is characterized by its location on the lattice and a number $f \in[0,1]$. All external conditions influencing the dynamics of the firms (labor conditions, fiscal system, availability of human and natural resources, etc) are summarized in a single value $F \in[0,1]$ - the external field. Since the agreement between $f$ and $F$ will determine the firm's survival, see below, we shall call $f$ the fitness of the firms.

Initially all firms are located at random positions in region I with initial density $c(0)$ and with random values of the $f$ 's. Like in the Bak and Sneppen extremal dynamics model [9] we choose a firm with the lowest appealing fitness from the field point of view, do not remove the firm immediately from the system as was done in the Bak and Sneppen model, but check the firm's survival chance. This depends on the difference between the fitness of the firm and the external field and the selection pressure. If the firm does not survive the check, it is removed from the system and all firms which are the nearest neighbors (von Neumann neighborhood) of the chosen firm receive new, random values for their fitness.

If not removed, the firm may change position, merges with another firm or creates spin-off(s).

After a certain time the border between the region I and region II is opened, thus permitting the motion of firms into regions II and III. At the same time the external field changes in region I, assuming a new value $F_{I}$, different from that in region II $\left(F_{I I}\right)$ and III $\left(F_{I I I}\right)$. There is only one such change of the field (in contrast to cases examined in [1]).

To complete a Monte Carlo Step (MCS) one has to pick as many firms 
as there were at the beginning of that step.

The algorithm goes as follows:

1. The firm $(i)$ which has its fitness farthest away from the field value is picked. The search is made in the whole system, and the fitness is compared to the region field value, i.e. the fitness of a firm in the region I is compared with $F_{I}$, that in the region II with $F_{I I}$, etc.

2. The survival probability is calculated as

$$
p_{i}=\exp \left(-s e l\left|f_{i}-F\right|\right)
$$

and checked against a random number $r_{i} \in[0,1]$ taken from a uniform distribution. If $r_{i}>p_{i}$, the firm is removed from the system and its nearest neighbors are given new, arbitrary, but within the $[0,1]$ interval, values of fitness,

3. If the firm survived, then 7 random searches are made in the von Neumann neighborhood (4 sites) for an empty place. As soon as the search is successful, the firm moves there.

4. A random search is next made for a partner in the von Neumann neighborhood of the new position. If found at the site $j$ then

5. with a probability 0.01 the two firms merge, creating a new firm at the location of the first one, with a new fitness given by

$$
f_{i}=\frac{1}{2}\left[\left(f_{i}+f_{j}\right)+\operatorname{sign}[0.5-r]\left|f_{i}-f_{j}\right|\right],
$$

where $r$ is a random number in $[0,1]$. The second firm is eliminated. If the new fitness for the (first) firm is greater than 1, the firm is eliminated too.

6. with a probability 0.99 the two firms produce a new $(k)$ firm (spin-off) with a fitness given by the formula

$$
f_{k}=\frac{1}{2}\left[\left(f_{i}+f_{j}\right)+\operatorname{sign}[0.5-r]\left|f_{i}-f_{j}\right|\right],
$$


15 random searches in the Moore neighborhood (9 sites) of the first firm are made to put the spin-off firm there at the first found empty site. If the attempts are unsuccessful, the new firm is not created.

After choosing all agents, one MC time step is done. We have in such a way limited our investigations to 1500 MCS.

In each case, we have started with an initial concentration $c=0.8$ in region I, and have destroyed the wall between region I and II after 100 MCS. The external field takes values $0.3,0.5,0.6$ in the three regions after $t_{1}$ and remains constant thereafter. The results reported in Sect. 3 refer to averages over ten runs.

\section{Results}

In the following we stress results pertaining to cases demonstrating the newness of the model with respect to our previous work [1].

In Fig. 1, the number of firms existing in the three regions is shown as a function of time for a sel $=0.5$. Recall that there are 10050 lattice sites in each region. It is observed that there is a rapid decrease in concentration in the first region as soon as the I-II wall is open, but the death and birth process stabilizes the concentration at nearly $c=0.4$ after a few Monte-Carlo steps, even though the concentrations in regions II and III still increase due to the largely available space.

It is unclear whether for very long time the concentrations would level off to the value of region I or to other values in regions II and III. However an interesting qualitative information is seen through the position of the diffusion front (going, recall from left to right, in this world) for various sel values. (Fig.2). The break toward some stationary-like asymptotic concentration value in region I, as seen in Fig. 1, seems to occur at the same time as the front progresses, as seen in Fig. 2; the break seems to mark an apparently different growth law. We have not studied the diffusion coefficient, but the difference in behavior might be traced to the effects of diffusion (together with the birth and death) process in presence of (moreover here mobile) barriers, as in $[19,20]$. The front propagation for sel $<<0.65$ seems to be a simple Brownian like process.

The complete spatial distribution and its evolution in time would require a movie like display; instead we give snapshots for the value of the concentra- 
tion along vertical columns, for a few Monte-Carlo times and two sel values in Fig. 3(a-b) respectively. Recall that sharp field gradients occur at $x=50$ and 100 . For the low sel $(=0.3)$ the concentration in region I hardly varies with time (and column position), and remains near 0.4, as can be hinted from Fig.1 for sel $=0.5$. After sufficient Monte-Carlo steps the concentration seems to level off in region II, although it is not clear what would be the final concentration at very large MC time. For "large" sel value the concentration in region I is markedly decreased, and is quasi nonexistent in regions II and III.

It is therefore of interest to emphasize the role of sel at an intermediary time, as in Fig. 4, where it is seen that the behavior does not much differ from low to high sel except for the concentration amplitude, - which seems to decrease linearly with sel in region I.

The fitness value evolution in the three regions is not spectacular since it "rather quickly" reaches the optimal one, as constrained by the external field. The fitness optimal value in each region is easily reached and remain stable with MCS. It is more interesting to display the behavior of the fitness in line with the Bak-Sneppen dynamics. Recall that the interest of the dynamics is, beside the entity interactions, the choice of the less fit for starting an avalanche process. Therefore we have calculated the number $(\mathrm{N})$ of times the less fit entity is reached (picked), and its fitness value, during the process. The data for $\mathrm{N}$ as a function of $f$ in the case $s e l=0.5$ is shown in Fig. 5 on a semi-log plot.

It is observed that the number of unfit entities is large and about the same in the three regions. Moreover a rather well defined gap occurs in the distribution of "picked firms", - a gap centered on the external field value. This $f_{\text {gap }}$ can be displayed in Fig. 6 as a function of sel. Except for the asymmetry due to the external field value, the behavior of the gap can be reconciled with what is expected for the density at liquid-gas transitions as a function of temperature. The finite size of the system is likely the cause for inducing a non zero gap at large sel. A critical sel value near 0.66 is found whatever the external field. 


\section{Conclusions}

In summary, we have adapted a birth-death-diffusion process of macroeconomic evolution with a Bak-Sneppen-like dynamics. From this set of results, we have observed that there are relatively well marked effects due to the "selection pressure", including a "critical value" reminiscent of the critical temperature at second order phase transitions. The constraining economic ("external") field implies stable concentration distributions, as far as examined. The diffusion process is not spectacular, apparently being Brownian like. We have not searched for avalanches as found in self-organized processes.

Result robustness should be further checked with respect to the parameters which are involved : number of regions, lattice size(s), lattice symmetry, initial concentration(s), field time sequence(s), and time for $F$ field changes, selection pressure, hopping distance for the diffusion process, number of 'spin off's, the latter creation probability, ...

Further improvements can be also suggested both from a macro and micro economy point of view, as well as from physical system studies. A company cannot be described by one scalar number $f_{i}$, but a vector model coupled to a vector field should be more realistic. Moreover the role of the business plan through the birth and death process description mapping merging and spin off processes is also to be improved. The fitness distribution and evolution law(s) might also be changed for better reflecting macroeconomy findings. Analytical work could be of interest to search for bifurcation and chaotic conditions, if any.

\section{Acknowledgments}

MA and AP thank the CGRI and $\mathrm{KBN}$ for partial financial support allowing mutual visits during this work process. MA and PC also thank an Action Concertée Program of the University of Liège (ARC 02/07-293). 


\section{References}

[1] M. Ausloos, P. Clippe, and A. Pȩkalski, Simple model for the dynamics of correlations in the evolution of economic entities under varying economic conditions, Physica A 324 (2003) 330

[2] H. E. Stanley, M. Ausloos, J. Kertész, R. N. Mantegna, J. A. Scheinkman and H. Takayasu, Preface of the International Scientific Organizing Committee, Physica A 324 (2003) xv

[3] P. Bak, How Nature Works: The science of self-organized criticality, (Oxford UP, Oxford, 1997)

[4] M. Kalecki, A Macrodynamic Theory of the Business Cycle, Econometrica 3 (1935) 327; M. Kalecki, A Theory of the Business Cycle, Rev. Econ. Studies 4 (1937) 77; M. Kalecki, Theory of Economic Dynamics: An essay on cyclical and long-run changes in capitalist economy, (Monthly Review Press, New York, 1965)

[5] A. Krawiec and M. Szydłowski, The Kaldor-Kalecki Model of Business Cycle, J. Nonlin. Math. Phys. 8 (2001) 266

[6] J. Miśkiewicz and M. Ausloos, Logistic map approach to economic cycle, submitted

[7] M. Ausloos, P. Clippe, and A. Pȩkalski, Model of macro-economic evolution in spatially dependent economic fields, submitted

[8] G.R. Richards, Reconciling econophysics with macroeconomic theory, Physica A 282 (2000) 325

[9] P. Bak and K. Sneppen, Punctuated equilibrium and criticality in a simple model of evolution, Phys. Rev. Lett. 71 (1993) 4083

[10] D. A. Head and G. J. Rodgers, The anisotropic Bak-Sneppen model, J. Phys. A 31 (1998) 3977

[11] N. Vandewalle and M. Ausloos, Self-organized criticality in phylogeneticlike tree growths, J. Phys. I France 5 (1995) 1011 
[12] M. Kramer, N. Vandewalle and M. Ausloos, Speciations and extinctions in a self-organized critical model of tree-like evolution, J. Phys. I France 6 (1996) 599

[13] N. Vandewalle and M. Ausloos, The screening of species in a Darwinistic tree-like model of evolution, Physica D 90 (1996) 262

[14] N. Vandewalle, H. Van Puyvelde, and M. Ausloos, Self-organized criticality can emerge even if the range of interactions is infinite, Phys. Rev. E 57 (1998) 1167

[15] O.V. Kovalev, Yu. M. Pis'mak, and V.V. Vechernin, Self-Organized Criticality in the Model of Biological Evolution Describing Interaction of "Coenophilous" and "Coenophobous" Species, Europhys. Lett. 40 (1997) 471

[16] R. Donangelo and H. Fort, A model for mutation in bacterial populations, Phys. Rev. Lett. 89 (2002) 38101

[17] G. Cuniberti, A. Valleriani, and J. L. Vega, Effects of regulation on a self-organized market, Quant. Fin. 1 (2001) 332

[18] T. Yamano, Regulation effects on market with Bak-Sneppen model in high dimensions, Intern. J. Mod. Phys. C 12 (2001) 1329

[19] A. Pȩkalski and M. Ausloos, Monte Carlo simulation of oxygen diffusion in planar model of 123 YBCO. Low Temperature Regime and Effect of Trapping Barrier, Physica C 226 (1994) 188

[20] A. Pȩkalski and M. Ausloos, Surface Tracer diffusion in the Presence of a Solid Barrier. A Monte-Carlo study, Surf. Sci. Lett. 344 (1995) L1271 


\section{Figure captions}

Figure 1 - Average (over 10 runs) number of firms existing in the three regions as a function of time for sel $=0.5$ and field values $F=0.3,0.5,0.6$ after $t_{1}=100 \mathrm{MCS}$.

Figure 2 - Position of the diffusion front for various sel values with external field values $F=0.3,0.5,0.6$ after $t_{1}=100 \mathrm{MCS}$ in the three regions respectively.

Figure 3 - The concentration in the three regions for a few MCS when the external field takes values $F=0.3,0.5,0.6$ after $t_{1}=100$ MCS for $\mathrm{sel}=$ (a) 0.3 and (b) 0.8 .

Figure 4 - The concentration in the three regions at $\mathrm{t}=1000 \mathrm{MCS}$, for external field values $F=0.3,0.5,0.6$ for different sel values.

Figure 5 - Semi-log plot for the number N of (picked) "less fit entities" (as defined in the text) in the three regions after 1500 MCS when the external field values $F=0.3,0.5,0.6$ respectively for $\mathrm{sel}=0.5$.

Figure 6 - Display of $f_{\text {gap }}$, i.e. gap in picked $f$ values, as a function of sel in the three regions when the external field values are $F=0.3,0.5,0.6$ respectively. 


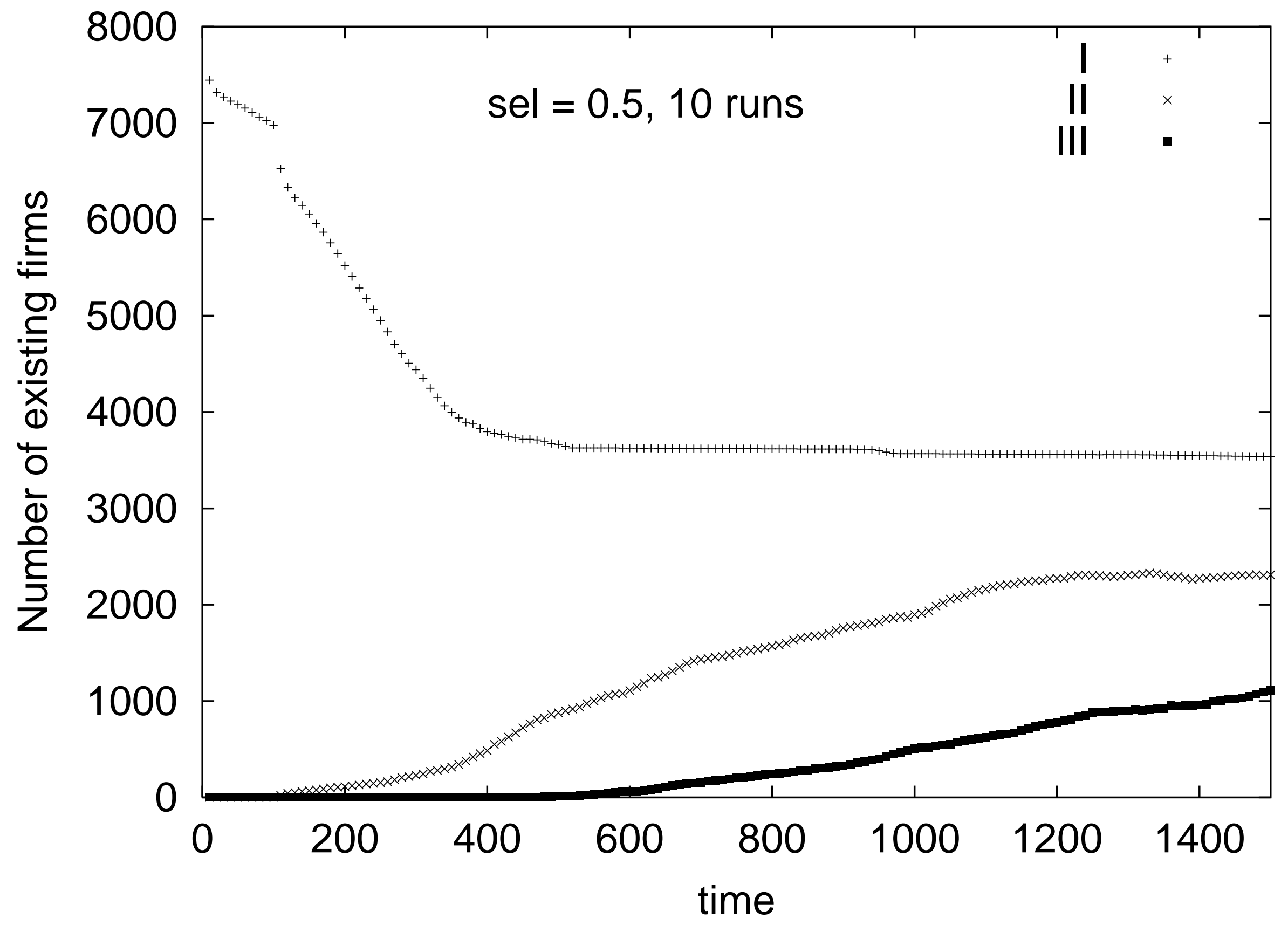

